\title{
Disks of Classical Be Stars
}

\author{
Stanislav Štefl \\ Astronomical Institute, Academy of Sciences, CZ-25165 Ondřejov, Czech Republic
}

\begin{abstract}
Observational methods used in investigation of circumstellar disks of Be stars significantly changed during the last decade. While mostly the studies of line profiles in the optical and near-IR region were used in the 70th and 80th, the present progress is done also thanks to interferometric and polarimetric observations. These techniques enable to determine the geometrical parameters of the circumstellar disks directly for the first time. They are flattened and axially symmetric. Their $\mathrm{H} \alpha$ emission region extends typically up to several tens of stellar radii. Systematic long-term spectroscopic monitoring helped to define and partly interpret the lineprofile variability of emission lines on different time-scales. Variations on a timescale of hours may appear after the matter is ejected during the outburst. The $V / R$ variations with periods of the order of 10 years reflect the global disk oscillations.
\end{abstract}

\section{Introduction}

Jaschek et al. (1980) defined a Be star as a non-supergiant B-type star, whose spectrum has, or had at some time, one or more Balmer lines in emission. Because some other definitions include also supergiant stars, the adjective "classical" was later added to the stars that meet the Jaschek's narrower definition.

The emission in optical, mainly Balmer and Fe II, lines and in the IR continuum originates in circumstellar disks around Be stars. In this paper, I attempt to summarize the recent results of observations of the disks and their main geometrical and physical properties. For more general reviews on Be stars or those directed to other aspects of Be stars see the proceedings of IAU Coll. 92 and IAU Symp. 162.

\section{Observations of Be star disks}

\subsection{Basic types of emission line profiles and their variations}

Extensive observations of the Bochum group in the southern hemisphere and several other groups in the north lead to the classification of the emission profiles of Be stars. Classification of the emission line spectra and their variability was discussed e.g. by Dachs (1986), Hanuschik et al. (1988). In Fig. 1, examples of the prototype emission profiles are given.

The type of an emission line profile correlates with $v$ sin $i$. The winebottle profile can be expected mainly for pole on stars, double-peak for medium 

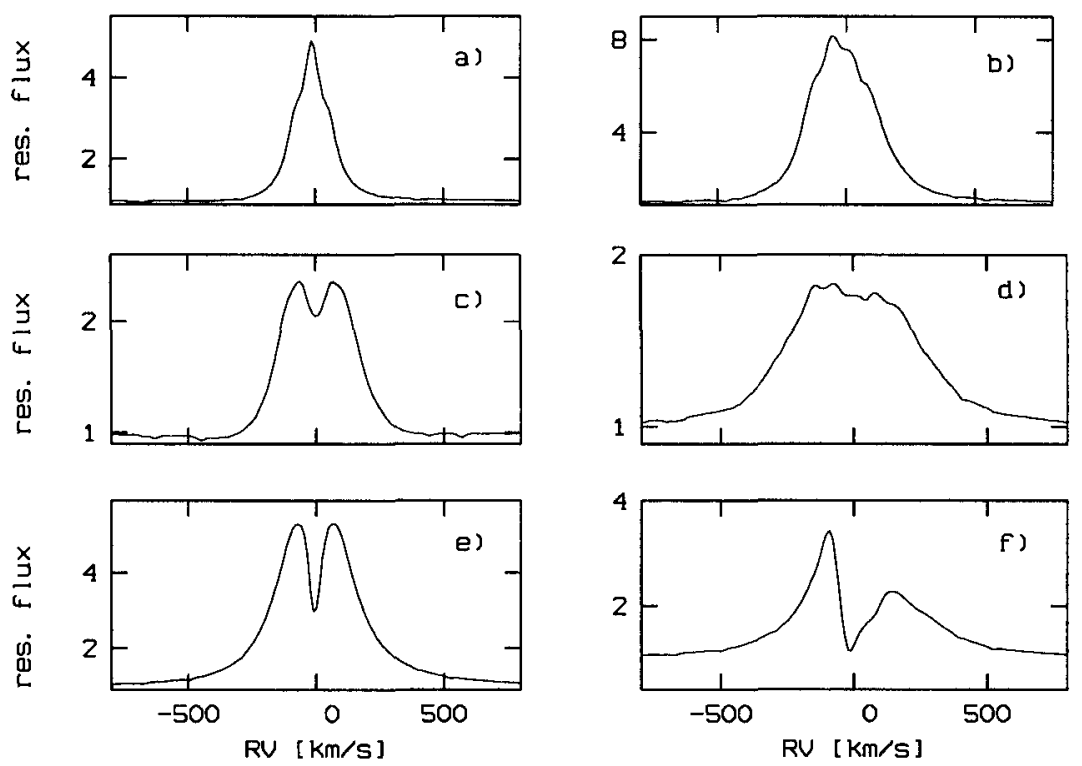

Fig. 1. The typical emission $\mathrm{H} \alpha$ profiles of Be stars: a) wine-bottle symmetric (11 Cam on March 1, 1997), b) wine-bottle asymmetric (66 Oph on June 24,1994), c) double-peak symmetric (23 Tau on October 11, 1995), d) double-peak asymmetric (59 Cyg on November 1, 1994), e) shell symmetric ( $\psi$ Per on March 1, 1997) and shell asymmetric ( $\zeta$ Tau on March 4, 1997). Profiles from the top downward demonstrate the effect of increasing inclination angle $i$. Asymmetric profiles (right column) are generally variable. The symmetric profiles are stable or can appear at a given phase of variable profiles. All spectra have been obtained with the $2 \mathrm{~m}$ telescope of the Ondřejov observatory.

inclination and shell profile for equator-on stars. However, the inclination is not the only determining parameter. The type can vary on a time-scale of years. This can be documented by the history of the first known Be star $\gamma$ Cas. During a short interval between 1930 and 1942, the type of the emission profile changed at least nine times and all types shown in Fig. 1 were observed in $\mathrm{H} \alpha-\mathrm{H} \delta$. In 1942, the emission even disappeared and a quasi-normal B type spectrum was observed for several months.

\subsection{Long-term $E / C$ and $V / R$ variations}

The $\mathrm{E} / \mathrm{C}$ variations, defined as the maximum $\mathrm{H} \alpha$ emission expressed in units of the continuum flux, take place on a time scale as long as 10-20 years. The time-scale is longer for later spectral types (Hubert-Delplace, 1981). 
The $V / R$ parameter is defined as the ratio of the flux in the violet and red peaks of the double peak emission line. Most frequently it is used for $\mathrm{H} \alpha$ profiles. Their V/R variations appear in about three quarters of Be stars. The quasi-periods are in the range of $2-22$ years and are strongly concentrated around 7 years. Lower amplitude variations on a time scale of tens of days - months may be superimposed, as in the case of $66 \mathrm{Oph}$ (Štefl et al., in preparation). The $\mathrm{V} / \mathrm{R}$ variability can cease for several years, as in $\gamma$ Cas for 25 years. The quasi-periods do not depend on the spectral type or $\mathrm{v} \sin \mathrm{i}$. They can change from cycle to cycle (e.g. 28 Tau: 22 and 6 years; Gulliver, 1977, Goraya and Tur, 1988). The shifts between V/R variations of different Balmer lines exclude the Huang's (1973) model of apsidal motion of material in an elliptical orbit. We suppose that the variations reflect the density waves in circumstellar disks (see Sect. 3).

\subsection{Transient periodicities}

The discovery and main properties of transient periodicities were reported by Stefl et al (1998). They were detected during the long-term monitoring of the Be stars $28 \mathrm{CMa}, \mu$ Cen, and $\eta$ Cen. The transient periodicities co-exist with the photospheric line-profile variations (periods $0.5-2$ days) and differ by $\approx 10 \%$ from them. They are present for a few days ( $\mu$ Cen) up to a few months (28 CMa). The transient periodicities are either not or only weakly detectable in photospheric lines, attain significant power only in lines formed (also) above the photosphere and are indicated mainly in the line wings. They do not re-appear with the same period as at the previous epoch. In the case of $\mu$ Cen, the transient periodicities excited during different outbursts fall in the interval $\pm 10 \%$. We suspect that transient periodicities reflect the processes in the region of star-disk interface, but this interpretation is more than preliminary.

\subsection{Rapid V/R variations}

Rapid V/R variations are observed on a time scale of hours to a few days. Rivinius et al. (1998a) published the study of $\mu$ Cen which contains probably the most detailed observations of the phenomenon. The $V / R$ variations in the wings of He I lines have a temporary character. They appear within 1 - 3 days after the outburst, last for 5-10 days and then $V / R$ converges to 1. Periods from different emission episodes differ up to $10 \%$. Very preliminarily, the authors interpret the variations as a cloud ejected during the outbursts and merging into the disk. Rapid V/R variations may reflect the same phenomenon as transient periods.

\subsection{Infrared, interferrometric and polarimetric observations}

The [12]-[60] vs. [12]-[25] colour diagram is consistent with free-free emission from the disk. IR excess decreases towards later spectral types (Gehrz et al., 
1974), but shows no clear correlation of IR excess vs. v sini. Observations indicate correlations of $\mathrm{V}$-[12] colour excess vs. spectral type and vs. $\mathrm{H} \alpha$ net emission.

The first star, for which the radius of the circumstellar disk was directly measured in the interferometric observations, was $\gamma$ Cas (Thom et al. 1986, Quirrenbach et al. 1993). The main results were achieved with the GI2T interferometer of the Observatoir de la Côte d'Azur and MKIII interferometer of the Mont Wilson Observatory. For more detailed and up-to-date new important results see the contributions by Stee and Vakili in these proceedings.

Be stars show a variable degree of polarization, but the polarization angle is constant. This indicates that the envelope is concentrated to a well defined plane and only negligible part of the polarization comes from the polar regions. Magnitude of polarization is wavelength dependent. It is lower in the lines and downward of Balmer discontinuity. It correlates with the $12 \mu \mathrm{m}$ IR excess (Coté and Waters, 1987) and $\mathrm{v} \sin \mathrm{i}$ (Brown and McLean, 1977). The position angle depends only on the disk orientation. For a more complete summary of the polarimetric results see K. Bjorkman (this Coll.).

\section{Geometrical and physical properties of the disks}

Thanks to the recent interferometric and polarimetric observations, the long lasting discussion about sphericity of the Be envelopes was solved. The flattened disks are confirmed both by direct interferometric observations and by the correlation of interferometric and polarimetric positional angles. Their differences are very close to $90^{\circ}$ in accordance with the model predicting scattering mostly in the equatorial plane of the disk.

The angular radii $a$ and flattening $r$ of the selected Be stars in $\mathrm{H} \alpha$ were measured interferometrically by Quirrenbach et al. (1997). Their diameters derived with the help of Hipparcos distances are listed in Tab. 1. Although there are still only few measured stars for a statistics, a typical diameter of the circumstellar disk seems to be 20 - 30 stellar diameters. Thus, the interferometric observations confirmed typical diameters of the disks $\left(\approx 20 R_{\star}\right)$ found by Hanuschik (1986), who analyzed the $\mathrm{H} \alpha$ emission profiles and applied the rotating disk model.

The diameter of the disk depends on the spectral line selected for the interferometric measurements. The VLA interferometry of $\psi$ Per done at $15 \mathrm{GHz}$ (Dougherty and Taylor, 1992) gives the angular diameter of $111 \pm 16$ mas, it is 34 times the diameter measured in $\mathrm{H} \alpha$. The positional angles from $\mathrm{H} \alpha$ and radio observations agree within the errors $\left[(-33 \pm 11)^{\circ}\right.$ and $(-32 \pm 10)^{\circ}$, respectively]. This indicates that the disk plane is well defined up to a distance of several hundred of stellar diameters.

The inner radii of the disks were not measured so far. The spectroscopic observations indicate that they are very different for different stars, change 
Table 1. Interferometrically measured geometrical parameters of Be star disks: $R_{\star}$ - equatorial stellar radius (Harmanec, 1988), a - angular diameter, $r$ - flattening (Quirrenbach et al., 1997), $\mathrm{d}_{\mathrm{Hip}}$ - Hipparcos distance (Perryman et al., 1997), D disk diameter in solar and in stellar radius.

\begin{tabular}{||l|l|c|c|c|r|r|r||}
\hline Star & $\begin{array}{l}\text { Spect. } \\
\text { type }\end{array}$ & $\begin{array}{c}\mathrm{R}_{\star} \\
{\left[\mathrm{R}_{\odot}\right]}\end{array}$ & $\begin{array}{c}\mathrm{a} \\
{[\mathrm{mas}]}\end{array}$ & $\mathrm{r}$ & $\begin{array}{r}\mathrm{d}_{\mathrm{Hip}} \\
{[\mathrm{pc}]}\end{array}$ & $\begin{array}{c}\mathrm{D} \\
{\left[\mathrm{R}_{\odot}\right]}\end{array}$ & $\begin{array}{c}\mathrm{D} \\
{\left[\mathrm{R}_{\star}\right]}\end{array}$ \\
\hline \hline$\gamma$ Cas & B0 IVe & 5.21 & $3.47 \pm .02$ & $0.70 \pm .02$ & 188 & 140 & 27 \\
$\phi$ Per & B1 Vep & 4.63 & $2.67 \pm .20$ & $0.46 \pm .04$ & 219 & 126 & 27 \\
$\psi$ Per & B5 Ve & 2.95 & $3.26 \pm .23$ & $0.47 \pm .11$ & 215 & 151 & 51 \\
$\eta$ Tau & B7 IIIe & 8.2 & $2.65 \pm .14$ & $0.95 \pm .22$ & 113 & 64 & 8 \\
48 Per & B3 Ve & 3.56 & $2.77 \pm .56$ & $0.89 \pm .13$ & 170 & 101 & 28 \\
$\zeta$ Tau & B4 IIIpe & $\approx 5$ & $4.53 \pm .52$ & $0.28 \pm .02$ & 128 & 125 & 25 \\
$\beta$ CMi & B8 Ve & 2.43 & $2.65 \pm .10$ & & 52 & 30 & 12 \\
\hline \hline
\end{tabular}

in the given star with its emission activity and may even approach the stellar photosphere.

The opening angel $\alpha$ cannot be derived unambiguously, because it is impossible to separate the inclination and thickness of the disks. The published results are inconsistent. Hanuschik (1996) derived $\alpha \approx 13^{\circ}$ from the observed number of shell stars, Waters et al. (1987) $\alpha \approx 15^{\circ}$ by modelling the IR excess. However, the wind compressed model of Bjorkman and Cassinelli (1993) requires $\alpha \leq 3^{\circ}$.

The tilt angle $\epsilon$ between rotational axes of the star and the disk was assumed to be zero. Only recently Hummel (1998) published his study of the Be stars $\gamma$ Cas and $59 \mathrm{Cyg}$, in which he breaks this taboo. Assuming a non-zero tilt angle increasing as the emission episodes develop, he could roughly model observed I) variations of the emission-line type, II) line width variations and III) long-term brightness variations. His model suggests a nonzero tilt angel only as a temporal effect in one emission episode for each of the two studied Be stars, but not as a general property. The model also does not explain the physical origin of the tilt. According to Porter (1998), the Be star disks may develop radiatively induced warps in their inner regions. It is possible that the effects explained by Hummel as variable tilt of the disk are in fact manifestations of the warps. The time-scale predicted for the warps is consistent with that derived by Hummel.

The densities of circumstellar disks were derived mainly from the IR excess (Waters et al. 1987) and by modeling the asymmetric UV lines (Snow, 1981). The former method indicates the relation $\rho(r)=\rho_{0}\left(r / R_{\star}\right)^{-n}$ with $\log \rho_{0}=-(11.0-11.6) \mathrm{g} / \mathrm{cm}^{3}$ and $2.0 \leq n \leq 3.5$. The mass-loss $\dot{\mathrm{M}}_{I R} \approx$ $10^{-8} \mathrm{M}_{\odot} /$ year and depends only weakly on luminosity. The latter method gives the mass-loss $\dot{\mathrm{M}}_{U V} \approx 10^{-10} \mathrm{M}_{\odot} /$ year and it depends strongly on luminosity. Comparison of the both methods indicates that the mass-loss is strongly asymmetric and that the mechanisms responsible for the mass loss in the infrared and ultraviolet may be different. 


\section{Dynamical structure of the disks}

The Be star disks are quasi-Keplerian. Okazaki (1991) found that global (socalled one-armed) oscillations can proceed in the disks in a retrograde way. Papaloizou et al. (1992) and Savonije and Heemskerk (1993) derived that prograde modes can be excited by perturbation from a rotationally flattened rapidly rotating star. In the Okazaki's (1997) hybrid scenario, the one armed oscillation can occur in the disk due to perturbation by radiation pressure and rotationally distorted star. The former effect is dominant for early $\mathrm{Be}$ stars (B0 - B3), the latter for the later Be stars.

The existence of one armed oscillations is supported by the following observational facts:

- predicted periods agree with the observations (Okazaki, 1997)

- line profiles calculated for $m=1$ perturbation agree with observed line profile variability (Hummel and Hanuschik, 1997)

- appearance of UV absorption components in $\gamma$ Cas is higher when V/R $\geq 1$ (Telting and Kaper, 1994)

- Observed correlation between $\mathrm{V} / \mathrm{R}$ and long-term photometric variations (Mennickent et al., 1997) is consistent with the model of global oscillations and seems to support existence of prograde modes.

- in the hybrid scenario, the period decreases with the increasing value of radiative parameter for earlier spectral types and with larger deformation parameter towards later spectral types. As the result, the maximum in period vs. spectral type distribution should occur around B3. The statistics of the observed periods may agree with this prediction, although the scatter in observed periods is large and correlation by far not convincing (see Okazaki, 1997).

\section{Formation of the Be star disks}

The physical process responsible for the formation of disks is not known even after many decades of intensive studies of these objects and we cannot exclude that more than one mechanism is involved.

It seems that the disks are formed mainly by sudden emission outbursts. Rivinius et al. (1998b) showed that the emission outbursts in the Be star $\mu$ Cen coincide with times of beating of photospheric periods derived from mode and line profile variations. Mass flow into the disk is then concentrated in relatively short time intervals after the outbursts.

In some stars, the continuous mass outflow from the photosphere plays an important role in supplying material into disks. However, the formation of disks by the wind compressed model (Bjorkman and Cassinelli, 1993; Owocki et al., 1994) meets serious theoretical and observational problems.

Acknowledgement: The author thanks Drs. D. Baade, W. Hummel and Th. Rivinius for valuable comments on the manuscript. 


\section{References}

Bjorkman J.E., Cassinelli J.P., 1993, ApJ 409, 429

Brown J.C., McLean I.S., 1977, A\&A, 57, 141

Coté J., Waters L.B.F.M., 1987, A\&A 176, 93

Dachs J., 1986, in IAU Coll. 92, p. 149

Dougherty S.M., Taylor A.R., 1992, Nature 359, 808

Gehrz R.D., Hackwell J.A., Jones T.W., 1974, ApJ 191, 675

Goraya P.S., Tur N.S., 1988, A\&A 205, 164

Gulliver A.F., 1977, ApJS 35, 441

Harmanec P., 1988, Bull. Astron. Inst. Czechosl. 39, 329

Hanuschik R., 1986, A\&A 166, 185

Hanuschik R.. 1996, A\&A 308, 170

Hanuschik R., Kozok J.R., Kaiser D., 1988, A\&A 189, 147

Huang S.S., 1973, ApJ 183, 541

Hubert-Delplace A.M., Jaschek M., Hubert H., Chambon M.Th., 1981, in IAU Symp. 98, p. 125

Hummel W., 1998, A\&A 330, 243

Hummel W., Hanuschik R., 1997, A\&A 320, 852

Jaschek M., Hubert-Delplace A.-M., Hubert H., Jaschek C., 1980, A\&AS 42, 103

Mennickent R.E., Sterken C., Vogt N., 1997, A\&A 326, 1167

Okazaki A.T., 1991, 1991, PASJ 42, 75

Okazaki A.T., 1997, A\&A 318, 548

Owocki S., Cranmer S.R., Blondin J.M., 1994, ApJ 424, 887

Papaloizou J.C., Savonije G.J., Henrichs H.F., 1992, A\&A 265, L45

Perryman M.A.C., Høg E., Kovalewski J., Lindegren L., Turon C., 1997, ESA SP1200, The Hipparcos and Tycho Catalogues

Porter J.M., 1998, A\&A 336, 966

Quirrenbach A., Hummel C.A., Buscher D.F., Amstrong J.T., Mozurkewich D., Elias II N.M., 1993, ApJ 416, L25

Quirrenbach A., Bjorkman K.S., Bjorkman J.E., Hummel C.A., Buscher D.F., Armstrong J.T., Mozurkewich D., Elias II N.M., Babler B.L., 1997, ApJ 479, 477

Rivinius Th., Baade D., Štefl S., Stahl O., Wolf B., Kaufer A.: 1998a, A\&A, 333, 125

Rivinius Th., Baade D., Štefl S., Stahl O., Wolf B., Kaufer A., 1998b, in Proc. A Half Century of Stellar Pulsation Interpretations: A Tribute to Arthur N. Cox, eds. P.A. Bradley and J.A. Guzik, Astron. Soc. Pacific Conf. Series, 135, 341

Savonije G.J., Heemskerk M.H.M., 1993, A\&A 276, 409

Snow T.P., 1981, ApJ 251, 139

Štefl S., Baade D., Rivinius Th., Kaufer A., Stahl O., Wolf B.: 1998, in Proc. $A$ Half Century of Stellar Pulsation Interpretations: A Tribute to Arthur N. Cox, eds. P.A. Bradley and J.A. Guzik, Astron. Soc. Pacific Conf. Series, 135, 348

Telting J.H., Kaper L., 1994, A\&A 284, 515

Thom C., Granes P., Vakili F., 1986, A\&A 165, L13

Waters L.B.F.M., Coté J., Lamers H.J.G.L.M., 1987, A\&A 185, 206 


\section{Discussion}

H. Lamers: What is the observational evidence that the disk is tilted with respect to the equator?

S. Śtefl: Hummel (1987) offers the hypothesis of non-zero and variable tilt angle and shows that it can explain quite well the long-term variations of the width of emission lines, brightness and the type of emission profiles. This makes the hypothesis attractive, but it is still not evidence that the disk is really tilted.

P. Stee: We have observed $\gamma$ Cas in He I $\lambda 6678$ and in the nearby continuum: we found that the emission comes from inner regions, e.g., from regions with $\mathrm{R} \leq 4 \mathrm{R}_{\star}$ (see Stee et al. 1998, A\&A 332, 268). Moreover, the He I $\lambda 6678$ emission comes from smaller regions than the $0.6 \mu \mathrm{m}$ continuum. These results are also confirmed by a recent paper by Moujtahid et al. (A\&A, in press) from photometric observations. This is in contradiction with WCD models or concave models (see Hanuschick et al.) where you have no matter very close to the star.

S. Shore: Given the similarity of the disks you are discussing to those of some binaries, such as $\omega$ Ser, $\beta$ Lyr, etc., what is known about the UV absorption in the systems you have discussed? In particular, models for $\omega$ Ser and other thick disk systems give strong Fe II/III, etc. absorption from the middle and outer disks. After all, whether binaries or single stars, one would expect that "disks are disks", right?

S. Śtefl: I am not aware of any significant observational difference between disks of $\omega$ Ser stars and classical Be stars. My personal opinion is that in general the subordinate UV absorption lines of Fe II are weaker in the disks of Be stars.

J. Bjorkman: UV polarisation observations of $\zeta$ Tau (a star for which interferometric observations indicate a thin disk) show large line blanketing around $1900 \AA$ and $2100 \AA$ indicating the presence of Fe III and Fe II in the disk, close to the star. So indeed the physical conditions in Be star disks are similar to the systems you mentioned.

A. Sapar: Has the fact that tilted disks must have a changing (perturbed) plane of revolution, as in the case of artificial earth satellites, been taken into account? Can this effect destroy the unique plane of the disk?

S. Sttefl: Yes, the effect of the perturbed plane of revolution was taken into account, but it is not necessary to explain the observations. This effect can really destroy the plane of the disk. This is indicated by the equation for the first order secular perturbation of the nodal position in Hummel (1998). 